\title{
Estereotipos sociales asociados a seis nombres propios en una muestra de cuarenta sujetos de nivel educacional superior (2)*
}

\author{
Gastón Salamanca Gutiérrez**, \\ Felipe Riffo Luengo***, \\ Daniela Riffo Mardonez****
}

\section{RESUMEN}

Este segundo artículo presenta los resultados obtenidos luego de aplicar el instrumento a sujetos de género masculino. Se concluye que, al igual que al aplicar el instrumento a sujetos de género femenino, existen estereotipos sociales muy definidos para cada uno de los nombres propuestos (Agustín, Marcelo y Jason (Yeison)). También, se concluye que las mujeres poseen mayor asertividad al momento de emitir sus juicios, lo que es compatible con los hallazgos de otras investigaciones en Sociolingüística.

Palabras clave: Estereotipos, nombres propios, Sociología del Lenguaje.

Social stereotypes associated to six names in a sample of forty subjects of higher education level (2)

\section{ABSTRACT}

This second article presents the results obtained after applying the instrument to male subjects. It concludes that, as in applying the instrument to female subjects, there are social stereotypes very defined for each of the proposed names (Augustín, Marcelo and Jason (Yeison)). It also concludes that women possess greater assertiveness when issuing their judgments, which supports the findings of other studies in Sociolinguistics.

Key words: Stereotypes, proper names, Sociology of Language.

* Este artículo constituye la segunda parte del estudio denominado "Estereotipos sociales asociados a seis nombres propios en una muestra de cuarenta sujetos de nivel educacional superior", el cual fue publicado en Foro Educacional No23.

Gastón Salamanca es Doctor en Lingüística, académico de la Facultad de Humanidades y Arte de la Universidad de Concepción. E-mail: gaston.salamanca@gmail.com

Felipe Riffo Luengo es Licenciado en Educación con Mención en Español por la Universidad de Concepción. E-mail: f.riffoluengo@gmail.com

Daniela Riffo Mardonez es Licenciada en Educación con Mención en Español por la Universidad de Concepción. E-mail: daniriffo@udec.cl 


\section{Introducción}

Este artículo contiene la segunda parte y final del trabajo sobre los estereotipos sociales asociados a seis nombres propios en una muestra de cuarenta sujetos de nivel educacional superior. El objetivo específico que se propone es determinar el grado de asociación que I9 sujetos de género masculino establecen entre los nombres Agustín, Marcelo y Jason y las variables sociales "apariencia", "gustos", "residencia” y "estudios". El marco de referencia (que incluye los conceptos de actitud lingüística, estereotipos lingüísticos y prestigio y estigmatización), la metodología (que incluye el instrumento aplicado y la muestra), y las consideraciones previas para el análisis y los resultados coinciden con los que se presentan en el primer artículo y se pueden ver en detalle en él ${ }^{1}$.

\section{Tabulación de las respuestas dadas por sujetos de género masculino}

\subsection{Tabulación de la categoría "APARIENCIA"}

\subsubsection{Sub-categoría "ESTATURA"}

\begin{tabular}{|c|c|c|c|}
\hline AGUSTÍN & & ESTATURA & \\
\hline & ALTA & MEDIANA & BAJA \\
\hline & $6(60 \%)$ & $3(30 \%)$ & $\mathrm{I}(10 \%)$ \\
\hline MARCELO & & ESTATURA & \\
\hline & ALTA & MEDIANA & BAJA \\
\hline & $2(18 \%)$ & $7(64 \%)$ & $2(18 \%)$ \\
\hline JASON & & ESTATURA & \\
\hline & ALTA & MEDIANA & BAJA \\
\hline & I $(20 \%)$ & I $(20 \%)$ & $3(60 \%)$ \\
\hline
\end{tabular}

Con el fin de facilitar la comprensión de la forma en que se obtienen los datos específicos para este artículo, en Anexo se presenta nuevamente la encuesta aplicada a los sujetos de género masculino. 


\section{Comentarios}

La estatura despertó un interés relativo entre los encuestados, lo que se aprecia en el hecho de que de los diecinueve jóvenes, diez señalaron aspectos de la estatura para el nombre Agustín, once para el nombre Marcelo, y cinco pare el nombre Jason. En esta sub-categoría, los atributos mencionados fueron: "(estatura) Alta”, "(estatura) Mediana” y "(estatura) Baja”.

En el caso del nombre Agustín, es interesante observar que la mayoría de los encuestados lo asoció con "(estatura) Alta”, con un $60 \%$; un $30 \%$, con "(estatura) Mediana", y solo un I0\% con "(estatura) Baja”. En el caso del nombre Marcelo, "(estatura) Alta” fue indicada solo por un I8\% de los encuestados, en tanto que "(estatura) Mediana” alcanzó un 64\%, lo que corresponde a la amplia mayoría de menciones en comparación con los otros dos atributos, y "(estatura) Baja” fue señalada por un I8\%, al igual que en el caso de "(estatura) Alta". Finalmente, en el caso del nombre Jason, es interesante constatar que sólo un 20\% señaló "(estatura) Alta" y "(estatura) Mediana”, mientras que un 60\% señaló "(estatura) Baja”, lo cual corresponde a la amplia mayoría de las menciones.

Nótese el descenso sistemático de los porcentajes del atributo "(estatura) Alta" desde Agustín hasta Jason, y el ascenso sistemático de los porcentajes del atributo "(estatura) Baja”, en ese mismo orden.

\subsubsection{Sub-categoría "CONTEXTURA"}

\begin{tabular}{|c|c|c|c|}
\hline \multirow{2}{*}{ AGUSTÍN } & & \multicolumn{2}{|c|}{ CONTEXTURA } \\
\hline & GRUESA & MEDIANA & DELGADA \\
\hline & $\mathrm{I}(\mathrm{I} 0 \%)$ & $\mathrm{I}(\mathrm{I} \% \%)$ & $8(80 \%)$ \\
\hline MARCELO & & \multicolumn{2}{|c|}{ CONTEXTURA } \\
\hline & GRUESA & MEDIANA & DELGADA \\
\hline & $4(50 \%)$ & $\mathrm{I}(\mathrm{I} 2 \%)$ & $3(38 \%)$ \\
\hline JASON & & \multicolumn{2}{|c|}{ CONTEXTURA } \\
\hline & GRUESA & MEDIANA & DELGADA \\
\hline & $0(0 \%)$ & $2(19 \%)$ & $9(8 \mathrm{I} \%)$ \\
\hline
\end{tabular}




\section{Comentarios}

La contextura, también, despertó un interés relativo entre los encuestados. En efecto, el nombre Agustín recibió diez menciones; el nombre Marcelo, ocho; y el nombre Jason, once. En esta sub-categoría, los encuestados mencionaron tres atributos: "(contextura) Gruesa”, “(contextura) Mediana” y "(contextura) Delgada”.

Para el caso del nombre Agustín, es interesante constatar que solo un $10 \%$ de los encuestados mencionó “(contextura) Gruesa” y "(contextura) Mediana”; la amplia mayoría, en tanto, asoció este nombre con una persona de contextura delgada, con un $80 \%$ de las menciones. En el caso del nombre Marcelo, el 50\% lo asoció con una persona de contextura gruesa, un 38\% con una persona de contextura delgada, y un $12 \%$ con una persona de contextura mediana. Finalmente, en el caso del nombre Jason, rompiendo en alguna medida con las expectativas, ninguno de los encuestados se inclinó por "(contextura) Gruesa"; un 19\% lo asoció con contextura mediana; y un 81\% mencionó "(contextura) Delgada”.

Resulta interesante constatar que en el caso de los nombres Agustín y Jason la contextura delgada alcanzó un $80 \%$ y un $81 \%$, respectivamente. Sería interesante indagar en las razones que los sujetos tuvieron para asociar mayoritariamente un nombre prestigioso, como Agustín, y uno estigmatizado, como Jason, con contextura delgada, pues en este plano sí se pueden producir diferencias importantes.

\subsubsection{Sub-categoría “COLOR DE PELO"}

\begin{tabular}{|c|c|c|c|c|c|}
\hline \multirow{2}{*}{ AGUSTIIN } & & \multicolumn{2}{|c|}{ COLOR PELO } & \multirow[b]{2}{*}{ PELIRROJO } & \multirow[b]{2}{*}{ TEÑIDO } \\
\hline & RUBIO & CASTAÑO & $\begin{array}{l}\text { NEGRO U } \\
\text { OSCURO }\end{array}$ & & \\
\hline & $4(50 \%)$ & $3(37 \%)$ & $\mathrm{I}(\mathrm{I} 3 \%)$ & $0(0 \%)$ & $0(0 \%)$ \\
\hline \multirow[t]{3}{*}{ MARCELO } & & \multicolumn{2}{|c|}{ COLOR PELO } & & \\
\hline & RUBIO & CASTAÑO & $\begin{array}{l}\text { NEGRO U } \\
\text { OSCURO }\end{array}$ & PELIRROJO & TEÑIDO \\
\hline & $0(0 \%)$ & $2(40 \%)$ & $3(60 \%)$ & $0(0 \%)$ & $0(0 \%)$ \\
\hline \multirow[t]{3}{*}{ JASON } & & $\begin{array}{c}\text { COLOR } \\
\text { PELO }\end{array}$ & & & \\
\hline & RUBIO & CASTAÑO & $\begin{array}{l}\text { NEGRO U } \\
\text { OSCURO }\end{array}$ & PELIRROJO & TEÑIDO \\
\hline & $0(0 \%)$ & $0(0 \%)$ & I (I00\%) & $0(0 \%)$ & $0(0 \%)$ \\
\hline
\end{tabular}




\section{Comentarios}

El color de pelo fue mencionado en menor medida que las subcategorías anteriores. En efecto, en el caso del nombre Agustín, fue mencionado por ocho personas; en el caso del nombre Marcelo, por cinco; y en el caso del nombre Jason, solo por una persona. En esta sub-categoría, los encuestados focalizaron tres atributos: "(pelo) Rubio", "(pelo) Castaño" y "(pelo) Negro u Oscuro" ("Pelirrojo" y "(pelo) Teñido" fueron considerados solo en el caso de las mujeres.

Para el caso del nombre Agustín, es interesante observar que la mayoría señaló el atributo "(pelo) Rubio", con un 50\%; un 37\% lo asoció con "(pelo) Castaño"; y solo un I3\% lo asoció con "(pelo) Negro u Oscuro". Para el caso del nombre Marcelo, es interesante que los encuestados no consideraron el atributo "(pelo) Rubio"; el atributo “(pelo) Castaño” logró un 40\%, y "(pelo) Negro u Oscuro” fue mencionado por un $60 \%$, convirtiéndose en el atributo con el mayor número de menciones. Finalmente, es interesante observar que el nombre Jason se asoció exclusivamente con "(pelo) Negro u Oscuro" (I00\% de las menciones).

\subsubsection{Subcategoría "VESTIMENTA"}

\begin{tabular}{|c|c|c|c|c|c|c|}
\hline AGUSTÍN & & ROPA & & & & \\
\hline & CARA & $\begin{array}{c}\text { NADA } \\
\text { LLAMATIVA }\end{array}$ & FORMAL & $\begin{array}{c}\text { NI } \\
\text { FLAITE } \\
\text { NI CARA }\end{array}$ & $\begin{array}{c}\text { ROPA } \\
\text { ANCHA }\end{array}$ & $\begin{array}{c}\text { ROPA DE } \\
\text { MARCA }\end{array}$ \\
\hline MARCELO & I $(50 \%)$ & I $(50 \%)$ & $0(0 \%)$ & $0(0 \%)$ & $0(0 \%)$ & $0(0 \%)$ \\
\hline & CARA & $\begin{array}{c}\text { NADA } \\
\text { LLAMATIVA }\end{array}$ & FORMAL & $\begin{array}{c}\text { NI } \\
\text { FLAITE } \\
\text { NI CARA }\end{array}$ & $\begin{array}{c}\text { ROPA } \\
\text { ANCHA }\end{array}$ & $\begin{array}{c}\text { ROPA DE } \\
\text { MARCA }\end{array}$ \\
\hline JASON & $0(0 \%)$ & $0(0 \%)$ & I $(50 \%)$ & I (50\%) & $0(0 \%)$ & $0(0 \%)$ \\
\hline & CARA & $\begin{array}{c}\text { NADA } \\
\text { LLAMATIVA }\end{array}$ & FORMAL & $\begin{array}{c}\text { NI } \\
\text { FLITE } \\
\text { NI CARA }\end{array}$ & $\begin{array}{c}\text { ROPA } \\
\text { ANCHA }\end{array}$ & $\begin{array}{c}\text { ROPA DE } \\
\text { MARCA }\end{array}$ \\
\hline & $0(0 \%)$ & $0(0 \%)$ & $0(0 \%)$ & $\left.\begin{array}{c}2(67 \%) \\
0\end{array}\right)$ & I (33\%) \\
\hline
\end{tabular}

Recuérdese que "Pelirrojo" y "(pelo) Teñido" fueron mencionados solo por los sujetos de género femenino. 


\section{Comentarios}

La sub-categoría "Ropa" fue considerada de manera menos prominente si la comparamos con las respuestas de las mujeres y con las sub-categorías anteriores. En efecto, de las diecinueve personas encuestadas, solamente dos mencionaron la ropa en el caso de los nombres Agustín y Marcelo; mientras que tres lo hicieron para el caso del nombre Jason. En esta sub-categoría, los encuestados focalizaron seis atributos: "(ropa) Cara”, "(ropa) Nada llamativa”, "(ropa) Formal”, “(ropa) Ni flaite ni cara”, "(ropa) Ancha” y “(ropa) De marca”.

Para el caso del nombre Agustín, es interesante observar que las menciones se repartieron equitativamente entre "(ropa) Cara" y “(ropa) Nada llamativa”, con un 50\%. En el caso del nombre Marcelo, las menciones, también, se repartieron de manera equitativa, aunque con un 50\% para "(ropa) Formal", y otro 50\% para "(ropa) $\mathrm{Ni}$ flaite ni cara”. Finalmente, en el caso del nombre Jason, es interesante advertir que las menciones se repartieron entre "(ropa) Ancha”, con un $67 \%$, y "(ropa) De marca”, con un 33\%.

\subsubsection{Sub-categoría "COLOR DE PIEL"}

\begin{tabular}{|c|c|c|}
\hline \multirow{2}{*}{ AGUSTÍN } & \multicolumn{2}{|c|}{ PIEL } \\
\hline & MORENA & BLANCA \\
\hline & I $(33 \%)$ & $2(67 \%)$ \\
\hline MARCELO & PIEL \\
\hline & $4(67 \%)$ & BLANCA \\
\hline & MORENA & $2(33 \%)$ \\
\hline JASON & MORENA & BLANCA \\
\hline & $8($ IOO $\%)$ & $0(0 \%)$ \\
\hline
\end{tabular}

\section{Comentarios}

La sub-categoría "Color de Piel" fue considerada por un número mayor de personas encuestadas, en comparación con la sub-categoría anterior ("Ropa"), aunque no alcanza los resultados de "Contextura" y "Estatura". En efecto, de las diecinueve personas encuestadas, en el caso del nombre Agustín, tres personas mencionaron el color de piel 
como un aspecto relevante de la apariencia; en el caso del nombre Marcelo, seis personas consideraron este aspecto; y en el caso del nombre Jason, ocho personas lo consideraron relevante. En esta sub-categoría, los encuestados mencionaron dos atributos: "(piel) Morena” y "(piel) Blanca”.

En el caso del nombre Agustín, es interesante observar que un 67\% lo asoció con "(piel) Blanca", mientras que un 33\% lo asoció con "(piel) Morena”. Para el caso del nombre Marcelo, los resultados fueron inversos, pues un 67\% lo asoció con "(piel) Morena” y un $33 \%$ con "(piel) Blanca”. Finalmente, es interesante observar que el nombre Jason fue asociado en un I00\% con "(piel) Morena”.

\subsubsection{Sub-categoría "ASPECTO"}

\begin{tabular}{|c|c|c|c|}
\hline AGUSTÍN & \multicolumn{3}{|c|}{ ASPECTO } \\
\hline & LLAMATIVO & NORMAL & ORDENADO \\
\hline & $0(0 \%)$ & $\mathrm{I}(50 \%)$ & $\mathrm{I}(50 \%)$ \\
\hline MARCELO & & ASPECTO \\
\hline & LLAMATIVO & NORMAL & ORDENADO \\
\hline & $0(0 \%)$ & $5(83 \%)$ & $\mathrm{I}(17 \%)$ \\
\hline JASON & & ASPECTO \\
\hline & LLAMATIVO & NORMAL & ORDENADO \\
\hline & $0(0 \%)$ & I $(50 \%)$ & I $(50 \%)$ \\
\hline
\end{tabular}

\section{Comentarios}

En la categoría "Apariencia", también, fue considerada la sub-categoría "Aspecto", aunque no se releva con una cantidad prominente de menciones. En efecto, en el caso del nombre Agustín, esta sub-categoría fue considerada solo por dos personas; en el caso del nombre Marcelo, por seis; y en el caso del nombre Jason, por dos personas, al igual que en el caso del nombre Agustín. En esta sub-categoría, los encuestados mencionaron tres atributos: "(aspecto) Llamativo", "(aspecto) Normal” y "(aspecto) Ordenado".

Para el caso del nombre Agustín, las menciones se repartieron entre "(aspecto) Normal” y “(aspecto) Ordenado", con un 50\% para cada uno. En el caso del nombre Marcelo, los resultados fueron menos 
homogéneos, ya que "(aspecto) Normal" obtuvo un 83\% de las respuestas -convirtiéndose así en el atributo con mayor número de menciones- y "(aspecto) Ordenado” alcanzó el I7\% restante. Llama la atención, por último, y rompe en cierta medida con las expectativas, que los resultados del nombre Jason coincidan con los del nombre Agustín, pues el 50\% lo asoció con "(aspecto) Normal” y el otro 50\% con "(aspecto) Ordenado".

\subsection{Tabulación de la categoría "GUSTOS"}

\subsubsection{Sub-categoría "PASATIEMPOS"}

\begin{tabular}{|c|c|c|c|c|c|}
\hline AGUSTÍN & & & & & \\
\hline & CANTAR & BAILAR & SALIR & $\begin{array}{c}\text { TV O } \\
\text { CINE }\end{array}$ & LEER \\
\hline & $0(0 \%)$ & $0(0 \%)$ & I $(17 \%)$ & $2(32 \%)$ & $0(0 \%)$ \\
\hline & MANUALIDADES & COCINA & ENSENAR & AYUDAR & $\begin{array}{c}\text { INTERNET } \\
\text { O PC }\end{array}$ \\
\hline & $0(0 \%)$ & $0(0 \%)$ & $0(0 \%)$ & $0(0 \%)$ & $0(0 \%)$ \\
\hline & JUGAR & COMPRAR & POLOLEAR & ANIMALES & HABLAR \\
\hline & $0(0 \%)$ & $0(0 \%)$ & $0(0 \%)$ & I (I7\%) & I (I7\%) \\
\hline & TOCAR VIOLÍN & MOLESTAR & CHISMES & FIESTAS & TRABAJAR \\
\hline & I (I7\%) & $0(0 \%)$ & $0(0 \%)$ & $0(0 \%)$ & $0(0 \%)$ \\
\hline & ESTUDIAR & AUTOS & $\begin{array}{c}\text { POWER } \\
\text { RANGERS }\end{array}$ & VICIOS & LUJO \\
\hline
\end{tabular}

\begin{tabular}{|c|c|c|c|c|c|}
\hline MARCELO & & & & & \\
\hline & CANTAR & BAILAR & SALIR & TV O CINE & LEER \\
\hline & $0(0 \%)$ & $0(0 \%)$ & $3(33 \%)$ & $0(0 \%)$ & $0(0 \%)$ \\
\hline & MANUALIDADES & COCINA & ENSENAR & AYUDAR & $\begin{array}{c}\text { INTERNET } \\
\text { O PC }\end{array}$ \\
\hline & $0(0 \%)$ & $0(0 \%)$ & $0(0 \%)$ & $0(0 \%)$ & $0(0 \%)$ \\
\hline & JUGAR & COMPRAR & POLOLEAR & ANIMALES & HABLAR \\
\hline & $0(0 \%)$ & $0(0 \%)$ & $0(0 \%)$ & $0(0 \%)$ & $0(0 \%)$ \\
\hline & TOCAR VIOLÍN & MOLESTAR & CHISMES & FIESTAS & TRABAJAR \\
\hline & $0(0 \%)$ & I (II $\%)$ & I (II $\%)$ & $2(23 \%)$ & I (II $\%)$ \\
\hline & ESTUDIAR & AUTOS & $\begin{array}{c}\text { POWER } \\
\text { RANGERS }\end{array}$ & VICIOS & LUJO \\
\hline & I (II $\%)$ & $0(0 \%)$ & $0(0 \%)$ & $0(0 \%)$ & $0(0 \%)$ \\
\hline
\end{tabular}




\begin{tabular}{|c|c|c|c|c|c|}
\hline JASON & & & & & \\
\hline & CANTAR & BAILAR & SALIR & TV O CINE & LEER \\
\hline & $0(0 \%)$ & I $(14 \%)$ & I $(\mathrm{I} 4 \%)$ & $0(0 \%)$ & $0(0 \%)$ \\
\hline & MANUALIDADES & COCINA & ENSENAR & AYUDAR & $\begin{array}{c}\text { INTERNET } \\
\text { O PC }\end{array}$ \\
\hline & $0(0 \%)$ & $0(0 \%)$ & $0(0 \%)$ & $0(0 \%)$ & I $(\mathrm{I} 4 \%)$ \\
\hline & JUGAR & COMPRAR & POLOLEAR & ANIMALES & HABLAR \\
\hline & $0(0 \%)$ & $0(0 \%)$ & $0(0 \%)$ & $0(0 \%)$ & $0(0 \%)$ \\
\hline & TOCAR VIOLÍN & MOLESTAR & CHISMES & FIESTAS & TRABAJAR \\
\hline & $0(0 \%)$ & $0(0 \%)$ & $0(0 \%)$ & $0(0 \%)$ & $0(0 \%)$ \\
\hline & ESTUDIAR & AUTOS & $\begin{array}{c}\text { POWER } \\
\text { RANGERS }\end{array}$ & VICIOS & LUJO \\
\hline & $0(0 \%)$ & I (I4\%) & I (I4\%) & I (I4\%) & I (I4\%) \\
\hline
\end{tabular}

\section{Comentarios $^{3}$}

Como se observa, la sub-categoría "Pasatiempos" presenta una amplia gama de atributos. En el nombre Agustín, el pasatiempo "Tv o Cine" fue mencionado por un $32 \%$, número elevado, si consideramos que en los otros dos nombres (Marcelo y Jason) no fue mencionado. Mismo caso se aprecia en "Tocar Violín”, que en Agustín recibió un I7\% de las menciones y ninguna en los otros dos nombres. Finalmente, es interesante observar que en el caso del nombre Jason fueron considerados los pasatiempos "Bailar", "Vicios", "Lujo", "Autos" y "Power Rangers", con un I4\% cada uno, mientras que en Agustín y Marcelo estos no fueron mencionados.

\subsubsection{Sub-categoría "MÚSICA"}

\begin{tabular}{|c|c|c|c|c|c|}
\hline AGUSTÍN & & & & & \\
\hline & EN INGLÉS & POP & REGGEATON & ROMÁNTICA & EN ESPAÑOL \\
\hline & I $(9 \%)$ & $2(18 \%)$ & $0(0 \%)$ & $0(0 \%)$ & $0(0 \%)$ \\
\hline & BAILABLE & CHILENA & ROCK & CUMBIA & HIP HOP \\
\hline & $0(0 \%)$ & $0(0 \%)$ & $2(18 \%)$ & I $(9 \%)$ & $0(0 \%)$ \\
\hline & CLÁSICA & REGGAE & METAL & ELECTRÓNICA & $\begin{array}{c}\text { SIN } \\
\text { ESPECIFICAR }\end{array}$ \\
\hline & I (9\%) & I (9\%) & $0(0 \%)$ & I $(9 \%)$ & $2(18 \%)$ \\
\hline
\end{tabular}

3 Cabe señalar que si bien las actividades "trabajar" y "estudiar" podrían no ser consideradas como pasatiempos, sí fueron mencionadas por los encuestados en este contexto, por lo cual decidimos incluirlas. 


\begin{tabular}{|c|c|c|c|c|c|}
\hline MARCELO & & & & & \\
\hline & $\begin{array}{c}\text { EN } \\
\text { INGLÉS }\end{array}$ & POP & REGGEATON & ROMÁNTICA & EN ESPAÑOL \\
\hline & $0(0 \%)$ & $0(0 \%)$ & $0(0 \%)$ & $0(0 \%)$ & $0(0 \%)$ \\
\hline & BAILABLE & CHILENA & ROCK & CUMBIA & HIP HOP \\
\hline & $0(0 \%)$ & $0(0 \%)$ & I $(20 \%)$ & I $(20 \%)$ & $0(0 \%)$ \\
\hline & CLÁSICA & REGGAE & METAL & ELECTRÓNICA & $\begin{array}{c}\text { SIN } \\
\text { ESPECIFICAR }\end{array}$ \\
\hline & $0(0 \%)$ & $0(0 \%)$ & I $(20 \%)$ & $0(0 \%)$ & $2(40 \%)$ \\
\hline
\end{tabular}

\begin{tabular}{|l|c|c|c|c|c|}
\hline JASON & & & & & \\
\hline & EN INGLÉS & POP & REGGEATON & ROMÁNTICA & EN ESPAÑOL \\
\hline & $0(0 \%)$ & $0(0 \%)$ & $4(31 \%)$ & $0(0 \%)$ & $0(0 \%)$ \\
\hline & BAILABLE & CHILENA & ROCK & CUMBIA & HIP HOP \\
\hline & $0(0 \%)$ & $0(0 \%)$ & $0(0 \%)$ & $7(53 \%)$ & $\mathrm{I}(8 \%)$ \\
\hline & CLÁSICA & REGGAE & METAL & ELECTRÓNICA & $\begin{array}{c}\text { SIN } \\
\text { ESPECIFICAR }\end{array}$ \\
\hline & $0(0 \%)$ & $0(0 \%)$ & $0(0 \%)$ & $0(0 \%)$ & $\mathrm{I}(8 \%)$ \\
\hline
\end{tabular}

\section{Comentarios}

La sub-categoría "Música" fue mencionada por un número importante de personas encuestadas. En efecto, de los diecinueve jóvenes consultados, once la mencionaron para el nombre Agustín; cinco, para el nombre Marcelo; y trece para el nombre Jason.

Para el caso del nombre Agustín "(música) Pop”, "(música) Rock” y "(música) Sin Especificar" encabezan el porcentaje de menciones, con un I8\%. Le siguen "(música) en inglés”, "(música) Clásica”, "Reggae", "(música) Electrónica” y "Cumbia”, con un 9\%. Para el caso del nombre Marcelo, los atributos expuestos fueron "(música) Rock", "Cumbia” y "(música) Metal”, con un 20\% cada uno, y "Sin Especificar", con un 40\%. Como se observa, estos porcentajes son mucho más compactos y no tan variados como los anteriores. Finalmente, en el caso del nombre Jason, es interesante observar que los atributos mencionados fueron "Cumbia", con un 53\% "Reggeaton", con un 31\%; y "Hip Hop" y "Sin Especificar", con un 8\%.

Nótese que en el caso del nombre Jason, "Cumbia" y "Reggeaton" concentran el $84 \%$ de las menciones, mientras que en los otros nombres estos atributos solo representan el 20\% -en el nombre Marcelo- y el 9\% -en el nombre Agustín. 


\subsubsection{Sub-categoría "COMIDA"}

Como mencionamos en el artículo anterior, esta sub-categoría fue considerada por las mujeres, pero no por los hombres.

\subsection{Tabulación de la categoría "RESIDENCIA"}

\subsubsection{Sub-categoría "LUGAR DE RESIDENCIA"}

\begin{tabular}{|c|c|c|c|c|c|}
\hline AGUSTÍN & & & & & \\
\hline & Ciudad & Pueblo & Villa & Campo & Población \\
\hline & $\mathrm{I5}(94 \%)$ & $\mathrm{I}(6 \%)$ & $0(0 \%)$ & $0(0 \%)$ & $0(0 \%)$ \\
\hline MARCELO & & & & & \\
\hline & Ciudad & Pueblo & Villa & Campo & Población \\
\hline & $\mathrm{II}(79 \%)$ & $0(0 \%)$ & $0(0 \%)$ & $0(0 \%)$ & $3(2 \mathrm{I} \%)$ \\
\hline JASON & & & & & \\
\hline & Ciudad & Pueblo & Villa & Campo & Población \\
\hline & $6(50 \%)$ & $0(0 \%)$ & $0(0 \%)$ & $0(0 \%)$ & $6(50 \%)$ \\
\hline
\end{tabular}

\section{Comentarios}

La sub-categoría "Lugar de residencia" fue considerada por un número importante de personas encuestadas, en comparación con las otras descritas anteriormente. En efecto, de las diecinueve personas encuestadas, dieciséis la mencionaron para el nombre Agustín; catorce, para el nombre Marcelo; y doce, para el nombre Jason. En esta subcategoría, los encuestados se refirieron a cinco atributos: "Ciudad", "Villa", "Pueblo", "Campo" y "Población".

Para el caso del nombre Agustín, resulta interesante observar que una amplia mayoría lo relacionó con una residencia en la ciudad (94\%), y solo el 6\% lo vinculó con "Pueblo". En el caso del nombre Marcelo, un elevado 79\% de las personas encuestadas consideró que quienes llevan este nombre viven en la ciudad, mientras que un 2I \% consideró que viven en una población. Para el caso del nombre Jason, resulta interesante observar que los porcentajes se repartieron equitativamente entre "Ciudad" (50\%) y "Población" (50\%).

Nótese que el mayor número de menciones en todos los casos fue para "Ciudad", lo cual es particularmente claro en los nombres Agustín 
y Marcelo, con un $94 \%$ y $79 \%$, respectivamente. Por otro lado, se destaca que el nombre Agustín no tenga asociación con "Población" y que el nombre Jason la tenga en un 50\%.

\subsubsection{Sub-categoría "CLASE SOCIAL DEL SECTOR DE RESIDENCIA"}

\begin{tabular}{|c|c|c|c|}
\hline AGUSTÍN & & & \\
\hline & BAJA & MEDIA & ALTA \\
\hline & $0(0 \%)$ & $\mathrm{I}(8 \%)$ & $\mathrm{I} 2(92 \%)$ \\
\hline MARCELO & & & \\
\hline & BAJA & MEDIA & ALTA \\
\hline & $\mathrm{I}(8 \%)$ & $9(75 \%)$ & $2(17 \%)$ \\
\hline JASON & & & ALTA \\
\hline & BAJA & MEDIA & I $(6 \%)$ \\
\hline
\end{tabular}

\section{Comentarios}

La sub-categoría "Clase social del sector de residencia", también, tuvo una alta consideración. En efecto, en el caso del nombre Agustín, I3 sujetos la mencionaron; en el caso del nombre Marcelo, I2; y en el caso de Jason, I6. En esta sub-categoría, los encuestados mencionaron tres atributos: "(clase) Baja”, "(clase) Media” y "(clase) Alta”.

Para el caso del nombre nombre Agustín, resulta interesante observar que el atributo "(clase) Baja” no obtuvo ninguna mención; "(clase) Media" obtuvo un 8\%; mientras que "(clase) Alta” obtuvo un altísimo 92\%. En el caso del nombre Marcelo, la mayoría de los encuestados consideró que quienes llevan este nombre residen en sectores que pertenecen a la "(clase) Media" (75\%); "(clase) Alta" obtuvo un I7\%; y "(clase) Baja”, un 8\%. En el caso del nombre Jason, resulta interesante observar que el atributo "(clase) Baja” se eleva notoriamente $(63 \%)$ con respecto a los otros dos nombres, mientras que "(clase) Alta" desciende a un exiguo 6\%. 


\subsection{Tabulación de la categoría "ESTUDIOS"}

\subsubsection{Sub-categoría "NIVEL DE ESTUDIOS"}

\begin{tabular}{|c|c|c|c|c|}
\hline AGUSTÍN & & & & \\
\hline & Básico & Secundario & Superior & No estudia \\
\hline & $2(25 \%)$ & $0(0 \%)$ & $6(75 \%)$ & $0(0 \%)$ \\
\hline MARCELO & & & & \\
\hline & Básico & Secundario & Superior & No estudia \\
\hline & $0(0 \%)$ & $2(25 \%)$ & $6(75 \%)$ & $0(0 \%)$ \\
\hline JASON & & & & Superior \\
\hline & Básico & Secundario & Nostudia \\
\hline & $2(\mathrm{I} 5 \%)$ & $3(2 \mathrm{I} \%)$ & $3(2 \mathrm{I} \%)$ & $6(43 \%)$ \\
\hline
\end{tabular}

\section{Comentarios}

En el caso del nivel educacional de los sujetos asociados con los nombres Agustín, Marcelo y Jason, la cantidad de menciones es relativamente importante. En efecto, Agustín y Marcelo recibieron ocho menciones, mientras que el nombre Jason recibió I4. En esta sub-categoría los atributos mencionados fueron "(nivel) Básico", "(nivel) Secundario", "(nivel) Superior" y "No estudia”.

El nombre Agustín presenta "(nivel) Superior" como el nivel educacional mayoritario, con un elevado $75 \%$; en tanto que "(nivel) Básico" recibe el 25\% de las menciones restantes. Para el nombre Marcelo, el nivel que predomina, también, es "(nivel) Superior", el cual, al igual que en el caso anterior, obtiene un $75 \%$ de las menciones; la diferencia radica en que "(nivel) Secundario" obtiene el segundo lugar con un $25 \%$. El nombre Jason presentó un atributo más que los nombres anteriores: "No estudia"; los atributos "(nivel) Secundario" y "(nivel) Superior" alcanzaron tres preferencias cada uno, con un $2 \mathrm{I} \%$. Este último dato es interesante, porque se distancia de manera importante del 75\% que obtiene este atributo en los nombres Agustín y Marcelo. Otro dato a considerar es que la mayor cantidad de menciones en el caso del nombre Jason la obtuvo el atributo "No estudia" con un $43 \%$, mientras que en los otros nombres este atributo no obtuvo menciones. 


\subsubsection{Sub-categoría "TIPO DE ESTABLECIMIENTO"}

\begin{tabular}{|c|c|c|c|}
\hline AGUSTÍN & \multicolumn{2}{|c|}{ TIPO DE COLEGIO } & \\
\hline & PÚBLICO & PART SUV & PRIVADO \\
\hline & I (II\%) & $0(0 \%)$ & $8(89 \%)$ \\
\hline MARCELO & \multicolumn{2}{|c|}{ TIPO DE COLEGIO } & \\
\hline & PÚBLICO & PART SUV & PRIVADO \\
\hline & I (I7\%) & $4(64 \%)$ & I $(17 \%)$ \\
\hline JASON & \multicolumn{2}{|c|}{ TIPO DE COLEGIO } & \\
\hline & PÚBLICO & PART SUV & PRIVADO \\
\hline & $8(\mathrm{I} 00 \%)$ & $0(0 \%)$ & $0(0 \%)$ \\
\hline
\end{tabular}

\begin{tabular}{|c|c|c|c|}
\hline AGUSTÍN & \multicolumn{2}{|c|}{ TIPO DE UNIVERSIDAD } & INSTITUTO \\
\hline & TRADICIONAL & PRIVADA & PROFESIONAL \\
\hline & I (I7\%) & $5(83 \%)$ & $0(0 \%)$ \\
\hline MARCELO & \multicolumn{2}{|c|}{ TIPO DE UNIVERSIDAD } & INSTITUTO \\
\hline & TRADICIONAL & PRIVADA & PROFESIONAL \\
\hline & $4(80 \%)$ & I $(20 \%)$ & $0(0 \%)$ \\
\hline JASON & \multicolumn{2}{|c|}{ TIPO DE UNIVERSIDAD } & INSTITUTO \\
\hline & TRADICIONAL & PRIVADA & PROFESIONAL \\
\hline & $0(0 \%)$ & $0(0 \%)$ & $6(100 \%)$ \\
\hline
\end{tabular}

\section{Comentarios}

En el tipo de colegio, para el nombre Agustín, es destacable el amplio predominio del atributo "(colegio) Privado", con un $89 \%$; le sigue "(colegio) Público", con el restante II\%. Para el nombre Marcelo, el 66\% mencionó "(colegio) Particular subvencionado", mientras que "(colegio) Público" y "(colegio) Privado” presentaron un I7\%. En el caso del nombre Jason, es notable que las ocho personas (I00\%) indicaron "(colegio) Público”.

Por otro lado, algunos de los encuestados vincularon el "Tipo de Establecimiento" con un nivel de educación superior. En el caso del nombre Agustín, es interesante observar que un altísimo $83 \%$ lo asoció con "(universidad) Privada", mientras que el I7\% restante indicó "(universidad) Tradicional”. En el caso del nombre Marcelo, un 80\% mencionó "Universidad tradicional", y un 20\%, "Universidad 
Privada”. Finalmente, es destacable que en el caso del nombre Jason las seis personas (I00\%) mencionaron "Instituto Profesional".

\section{Conclusiones}

En primer lugar, podemos afirmar que los objetivos propuestos al comenzar este trabajo se cumplieron, pues se logró determinar el grado de asociación que los sujetos establecieron entre los nombres propios presentados y las variables sociales establecidas ("apariencia”, "gustos", "residencia” y "estudios").

Cabe mencionar que para cada uno de los nombres propuestos existían expectativas de asociación, planteadas en términos de lo que se puede etiquetar como un juicio de expertos, por el trabajo constante con la lengua y su vínculo con la sociedad de quienes los emitieron. Así, por ejemplo, en el caso del nombre Julieta, en la categoría "Apariencia", sub-categoría "Estatura", la expectativa de respuesta mayoritaria era "(estatura) Alta”; para el nombre Sandra, "(estatura) Mediana”; y para el nombre Shirley, "(estatura) Baja”. En el caso de los hombres, en la categoría "Apariencia", sub-categoría "Aspecto", las expectativas de respuesta eran: para el nombre Agustín, "(aspecto) Ordenado"; para el nombre Marcelo, "(aspecto) Normal”; y para el nombre Jason, "(aspecto) Llamativo”. En la gran mayoría de los casos, estas expectativas sintonizaron totalmente con las respuestas de los sujetos ( $85,7 \%$ en el caso de las mujeres y $69,2 \%$ en el caso de los hombres) y en los demás fueron cumplidas de forma parcial o, en menor medida, no se cumplieron.

Es importante subrayar que las subcategorías y atributos surgieron a partir de las respuestas espontáneas de los sujetos encuestados, los cuales solo contaban con la categoría general como motivador para sus asociaciones (esto explica por qué en algunas subcategorías el número de menciones fue escaso). Este punto, a nuestro juicio, debe ser relevado, porque informa que lo que el sujeto encuestado plasma en sus respuestas es aquello que está disponible de manera expedita en su conciencia sociolingüística y no solo aquello que está dentro de loque el investigador estima cubre las respuestas posibles (lo que ocurriría al elicitar dichas respuestas a través de una pregunta cerrada). 
Otro aspecto relevante que arrojó este estudio, y que coincide con los precedentes bibliográficos, es que las mujeres son más asertivas en sus respuestas, por lo que es posible afirmar que los sujetos del género femenino aplican de manera más categórica los estereotipos sociales, aunque los hombres de igual modo presentan un porcentaje importante. Esto reafirma lo dicho por Tassara (1992), quien señala que son las mujeres y los jóvenes quienes emiten de manera más drástica sus juicios sobre aspectos referidos a la lengua.

Por otra parte, resulta evidente que los sujetos encuestados tienen clara conciencia de que determinados nombres despliegan una serie de asunciones culturales que se les atribuyen a quienes los portan ${ }^{4}$. No es casual, en efecto, por citar solo algunos casos, que el $60 \%$ de los encuestados asocie el nombre Agustín con una estatura alta, mientras que el mismo porcentaje estime que la estatura de quien porta el nombre Jason es baja; que un 67\% asocie el nombre Agustín con sujetos cuyo color de piel es blanca, mientras que un $80 \%$ asocia el nombre Jason con un color de piel morena; que un $84 \%$ asocie el nombre Jason con cumbia y reggeaton, mientras que estos ritmos prácticamente no son nombrados cuando se pone en foco el nombre Agustín; que un 50\% asocie el nombre Jason con la residencia en una población, mientras que dicho lugar ni siquiera es mencionado cuando se trata del nombre Agustín.

En un ámbito tan contingente como el educacional, es interesante constatar que un $75 \%$ de los encuestados asocia el nombre Agustín con sujetos que han cursado o cursan estudios superiores y que ninguno plantee la posibilidad de que el sujeto que porta este nombre no estudie, mientras que en el caso del nombre Jason un $43 \%$ señala que no estudia. No es atribuible al azar, tampoco, que un $89 \%$ asocie el nombre Agustín con un colegio privado, mientras que el I00\% asocia el nombre Jason con un colegio público; que el 100\% de los sujetos asocie el nombre Jason con un instituto profesional y que nadie mencione un centro de estudios de este tipo cuando se focaliza el nombre Agustín (el 83\% lo asocia, en cambio, con una universidad 4 Ejemplificamos solo con nombres masculinos, porque son los que se ponen en foco en este
artículo. 
privada). Finalmente, en uno de los ítemes más elocuentes, el 92\% asocia el nombre Agustín con clase alta, mientras que el 63\% asocia el nombre Jason con clase baja.

Un punto central a considerar aquí es que estos estereotipos se configuran socialmente, entre otros factores, por las asimetrías sociales enormemente pronunciadas de un país como Chile. En este contexto, se puede visualizar que la discriminación que se produce desde las clases sociales más privilegiadas respecto de las menos privilegiadas no solo se manifiesta en aspectos tradicionalmente destacados como la apariencia física, origen étnico, vestimenta, lugar de residencia, uso de la lengua (en sus distintos niveles de análisis: fonético-fonológico, morfosintáctico, léxico-semántico, discursivo-pragmático), etc., sino también a través de los nombres propios. En este contexto, un punto al que se debe prestar atención es que estas actitudes pueden no ser solo anecdóticas o inocuas, sino que pueden estar asociadas a mayores o menores oportunidades en el devenir cotidiano. Así, por ejemplo, un sujeto que se llame Cristóbal o Martín, Julieta o Isidora puede tener oportunidades que un sujeto llamado Jason o Byron, Sharon o Shirley no llegue a tener (o no en la misma medida), por el solo hecho de llevar este nombre. En este sentido, se considera que estudiar los estereotipos asociados a los nombres propios es seguir descubriendo la sociedad particularmente asimétrica.

Por último, dos proyecciones que se plantean a partir de este estudio son las siguientes: a) realizar un estudio similar en el ámbito rural, para pesquisar similitudes y diferencias con el ámbito urbano; y b) realizar un estudio similar con rangos etarios inferiores (estudiantes de Enseñanza Básica y Enseñanza Media, por ejemplo), con el fin de aproximarse a los períodos en que se empiezan a manifestar y consolidar los estereotipos sociales visibles en el lenguaje.

\section{Referencias bibliográficas}

Aguirre, V. \& Mella, P. (2012). Prestigio y estigmatización de 30 nombres propios en 30 sujetos de nivel educacional superior (Universitario). (Tesis para optar al grado de Licenciado en Educación con Mención en Español). Universidad de Concepción, Concepción, Chile. 
Fasold, R. (1996). La sociolingüística de la sociedad. Introducción a la sociolingüística. Madrid: Visor Libros.

Figueroa, M. (201 I). El eje oclusión/fricción en el sistema sociofónico del castellano de Chile. (Tesis para optar al grado de Magíster en Lingüística). Universidad de Concepción, Concepción, Chile.

Lastra, Y. (I992). Sociolingüistica para bispanoamericanos. México: El Colegio de México.

Millán, M. \& Rodríguez, C. (20II). Prestigio y estigmatización de 30 nombres propios en 44 sujetos de estrato sociocultural bajo. (Tesis para optar al grado de Licenciado en Educación con Mención en Español). Universidad de Concepción, Concepción, Chile.

Moreno-Fernández, F. (1990). Metodología sociolingüística. Madrid: Gredos

Pereira, L. (2012). Prestigio y estigmatización de 30 nombres propios en 40 sujetos de estrato sociocultural medio-alto. (Tesis para optar al grado de Licenciado en Educación con mención en Español). Universidad de Concepción, Concepción, Chile.

Salamanca, G. \& Pereira, L. (2013). Prestigio y estigmatización de 60 nombres propios en 40 sujetos de nivel educacional superior. Universum, en prensa.

Silva-Corvalán, C. (200I). Sociolingüistica y pragmática del español. Washington D.C.: Georgetown University Press.

Sociedad Bíblica (2006). Santa Biblia. Letra Grande. Brasil: Sociedades Bíblicas Unidas.

Tassara, G. (1992). Actitudes lingüísticas ante la variación de /č/. Revista de Lingüística Teórica y aplicada, 30, 263-271.

Trapero, M. (1996). Sobre la capacidad semántica del nombre propio. El Museo canario $L I, 337-357$.

Valdivieso, H. (1983). Prestigio y estigmatización: Factor determinante en la enseñanza institucionalizada de la lengua materna. Revista de Lingüística Teórica y aplicada, 21, 137-I42. 


\section{Anexos}

\section{Encuesta}

Te agradecemos de antemano por responder esta encuesta, la cual es anónima y contribuye a la formulación de un seminario de título de pregrado.

\section{Carrera:}

Edad:

a) ¿Con cuáles de los siguientes nombres crees que tus amigos se reirían de ti o te molestarían?, yy con cuáles piensas que te considerarían más o serías más popular? Selecciona 6 para cada categoría. Marca aquéllos con los cuales crees que serías bien visto con un $\checkmark$ y aquéllos con los cuales crees que se reirían de ti, con una $\mathrm{X}$.

\begin{tabular}{|l|l|l|l|l|l|l|l|l|l|l|l|}
\hline Cristóbal & & Máximo & & Jason & & Héctor & & Tomás & & Jordan & \\
\hline Alonso & & Jeremy & & Andrés & & Rodrigo & & Kevin & & Joaquín & \\
\hline Malcom & & Alejandro & & Michael & & Justin & & Agustín & & Ignacio & \\
\hline Pablo & & León & & Claudio & & Mauricio & & Brian & & Sebastián & \\
\hline Daniel & & Byron & & Harry & & Felipe & & Marcelo & & Martín & \\
\hline
\end{tabular}

b) ¿Puedes proponer 2 nombres más para las dos categorías? (uno de ellos, si quieres, puedes tomarlo de la tabla anterior).

Me molestarían también con los nombres:

I) 2)

Sería bien visto también con los nombres:

I) 2)

a) ¿Cuáles de estos nombres relacionas con un nivel social alto y cuáles con un nivel social bajo? Selecciona 6 y ubícalos en cada columna según corresponda.

Nombres de hombres:

\begin{tabular}{|l|l|l|l|l|l|l|l|}
\hline Cristóbal & Daniel & León & Michael & Rodrigo & Tomás & Marcelo & Sebastián \\
\hline Alonso & Máximo & Byron & Claudio & Justin & Kevin & Jordan & Martín \\
\hline Malcom & Jeremy & Jason & Harry & Mauricio & Agustín & Joaquín & \\
\hline Pablo & Alejandro & Andrés & Héctor & Felipe & Brian & Ignacio & \\
\hline
\end{tabular}




\begin{tabular}{|l|l|}
\hline NIVEL ALTO & NIVEL BAJO \\
\hline I. & I. \\
\hline 2. & 2. \\
\hline 3. & 3. \\
\hline 4. & 4. \\
\hline 5. & 5. \\
\hline 6. & 6. \\
\hline
\end{tabular}

b) ¿Puedes proponer 2 nombres más para las dos categorías? (uno de ellos, si quieres, puedes tomarlo de las tablas desde las cuales escogiste los nombres anteriormente).

Relaciono, también, con estrato bajo, los nombres masculinos: I) 2)

Relaciono, también, con estrato alto, los nombres femeninos: I) 2)

a) A partir de la lista de nombres que a continuación aparecen, selecciona 6 y ordena según tu preferencia para colocarle a tus hijos, 3 para hombre y 3 para mujer. Además elige 6 nombres que NO le pondrías a tus hijos, 3 para hombre y 3 para mujer.

NOMBRES PARA HOMBRE

\begin{tabular}{|l|l|l|l|l|l|}
\hline Cristóbal & Máximo & Jason & Héctor & Tomás & Jordan \\
\hline Alonso & Jeremy & Andrés & Rodrigo & Kevin & Joaquín \\
\hline Malcom & Alejandro & Michael & Justin & Agustín & Ignacio \\
\hline Pablo & León & Claudio & Mauricio & Brian & Sebastián \\
\hline Daniel & Byron & Harry & Felipe & Marcelo & Martín \\
\hline
\end{tabular}

NOMBRES PARA MUJER

\begin{tabular}{|l|l|l|l|l|l|}
\hline Alejandra & Sharon & Fabiola & Ignacia & Martina & Claudia \\
\hline Isidora & Joselyn & $\mathrm{M}^{a}$ Paz & Carolina & Andrea & Melany \\
\hline Shirley & Sofía & Nayareth & Maryori & Cecilia & Carla \\
\hline Antonia & Agustina & Yamileth & Daniela & Britney & Julieta \\
\hline Josefa & Paulina & Matilde & Yaritza & Natalia & Melody \\
\hline
\end{tabular}




\section{IMPORTANTE:}

Debes poner el nombre que más te gusta en el $\mathrm{N}^{\circ}$ I de la primera tabla, y el que menos te gusta en el $\mathrm{N}^{\circ}$ I de la segunda tabla, y continuar de forma decreciente en las casillas que siguen hasta completar los 12 nombres.

\begin{tabular}{|l|l|}
\hline \multicolumn{2}{|l|}{ NOMBRES QUE PREFERIRÍA } \\
\hline Si fuese hombre & Si fuese mujer \\
\hline I. & I. \\
\hline 2. & 2. \\
\hline 3. & 3. \\
\hline
\end{tabular}

\begin{tabular}{|l|l|}
\hline \multicolumn{2}{|l|}{ NOMBRES QUE NUNCA ELEGIRÍA } \\
\hline Si fuese hombre & Si fuese mujer \\
\hline I. & I. \\
\hline 2. & 2. \\
\hline 3. & 3. \\
\hline
\end{tabular}

b) ¿Puedes proponer 3 nombres más que tú elegirías para colocarle a tus hijos y 3 que por ningún motivo lo harías? (uno de ellos, si quieres, puedes tomarlo de las tablas desde las cuales escogiste los nombres anteriormente).

Si mi hijo fuera hombre, también podría colocarle:

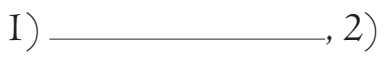
3)

Si mi hija fuera mujer, también podría colocarle:

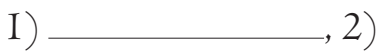
3)

Si mi hijo fuera hombre, por ningún motivo le pondría:<smiles>[3H]</smiles>
3)

Si mi hija fuera mujer, por ningún motivo le pondría:<smiles>[3H]</smiles>
3)

Imagina personas que lleven estos nombres y describe: a) su probable apariencia física, b) sus eventuales gustos, c) dónde vivirían y d) dónde estudiarían. 


\section{Agustín}

a)

b)

c)

d)

Marcelo

a)

b)

c)

d)

Jason

a)

b)

c)

d)

b) ¿Puedes proponer tú dos nombres más, que se ajusten a las descripciones que has hecho?

La descripción que hice para el nombre Agustín también valdría para los nombres

I)

La descripción que hice del nombre Marcelo también valdría para los nombres

I) y 2) 
La descripción que hice del nombre Jason también valdría para los nombres

$$
\text { I) }
$$

Finalmente: ¿Cuáles crees tú que son los factores que influyen para que un nombre sea considerado "bonito" o "feo"?

Para que sea considerado bonito:

Para que sea considerado feo:

¡Muchas gracias por tu colaboración! 